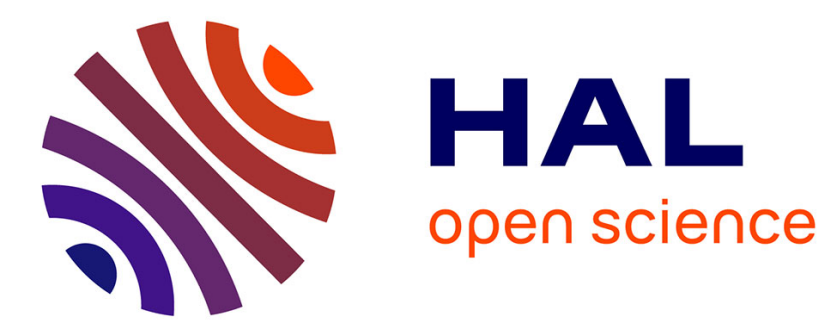

\title{
Light-Driven Transport of a Liquid Marble with and against Surface Flows
}

Nikita Kavokine, Manos Anyfantakis, Mathieu Morel, Sergii Rudiuk, Thomas Bickel, Damien Baigl

\section{- To cite this version:}

Nikita Kavokine, Manos Anyfantakis, Mathieu Morel, Sergii Rudiuk, Thomas Bickel, et al.. LightDriven Transport of a Liquid Marble with and against Surface Flows. Angewandte Chemie International Edition, 2016, 55 (37), pp.11183 - 11187. 10.1002/anie.201603639 . hal-01392406

\section{HAL Id: hal-01392406 https://hal.science/hal-01392406}

Submitted on 4 Nov 2016

HAL is a multi-disciplinary open access archive for the deposit and dissemination of scientific research documents, whether they are published or not. The documents may come from teaching and research institutions in France or abroad, or from public or private research centers.
L'archive ouverte pluridisciplinaire HAL, est destinée au dépôt et à la diffusion de documents scientifiques de niveau recherche, publiés ou non, émanant des établissements d'enseignement et de recherche français ou étrangers, des laboratoires publics ou privés.

\section{다(1)(2)}

Distributed under a Creative Commons Attribution - ShareAlikel 4.0 International 


\title{
Light-Driven Transport of a Liquid Marble with and against Surface Flows
}

\author{
Nikita Kavokine, Manos Anyfantakis, Mathieu Morel, Sergii Rudiuk, Thomas Bickel, and \\ Damien Baigl*
}

Abstract: Liquid marbles, that is, liquid drops coated by a hydrophobic powder, do not wet any solid or liquid substrate, making their transport and manipulation both highly desirable and challenging. Herein, we describe the light driven transport of floating liquid marbles and emphasize a surprising motion behavior. Liquid marbles are deposited on a water solution containing photosensitive surfactants. Irradiation of the solu tion generates photoreversible Marangoni flows that transport the liquid marbles toward UV light and away from blue light when the thickness of the liquid substrate is large enough (Marangoni regime). Below a critical thickness, the liquid marbles move in the opposite direction to that of the surface flow at a speed increasing with decreasing liquid thickness (anti Marangoni). We demonstrate that the anti Marangoni motion is driven by the free surface deformation, which propels the non wetting marble against the surface flow. We call this behavior "slide effect".

$T_{\text {he controlled transport of small amount of liquids is }}$ a crucial challenge for the improved performance of mini aturized devices with impact in diagnostics, healthcare, and energy. ${ }^{[1]}$ A robust and versatile strategy involves making fluids flow through microchannels ${ }^{[2]}$ but the confinement and contact of flowing fluids with the channel walls is a source of contamination and clogging. A channel free possibility involves the manipulation small volumes of fluids in the form of discrete drops deposited on a substrate. ${ }^{[3]}$ In this case, precise control of the wetting properties of the liquid to be transporte $\mathrm{d}^{[46]}$ is a challenge that tends to limit the applic ability of these strategies. To avoid all of the limitations due to specific liquid/substrate interactions (and especially contam ination and wetting issues), Aussillous and Quéré proposed a revolutionary idea. They coated water drops with hydro

[*] N. Kavokine, Dr. M. Anyfantakis, Dr. M. Morel, Dr. S. Rudiuk, Prof. Dr. D. Baigl

Ecole Normale Supérieure, PSL Research University

UPMC Univ Paris 06, CNRS, Department of Chemistry, PASTEUR

24 rue Lhomond, 75005 Paris (France)

and

Sorbonne Universités, UPMC Univ Paris 06

ENS, CNRS, PASTEUR 75005 Paris (France)

E mail: damien.baigl@ens.fr

Homepage: http://www.baigllab.com/

Dr. T. Bickel

Université de Bordeaux

Laboratoire Ondes et Matière d'Aquitaine, CNRS UMR 5798

33405 Talence (France) phobic particles and showed that the resulting structures, called liquid marbles, were in a non wetting situation regard less of their substrate. ${ }^{[7,8]}$ This result led to intense research to manipulate and understand the hydrodynamic behavior of these new entities. ${ }^{[9,10]}$

However, although a lot of progress has been made in the design and comprehension of liquid marbles, reported studies on their actuation have been limited to only a few cases. A typical strategy involves the use of stimuli responsive par ticles, including photosensitive ${ }^{[11,12]}$ and magnetic $\left.{ }^{[13} 15\right]$ parti cles suspended in or coating the marble, making remote actuation possible but making each strategy specific to the composition of the liquid marble. Composition independent approaches were restricted to the use of electric ${ }^{[8,16,17]}$ or ultrasonic ${ }^{[18]}$ stimulation but, in these cases, long range and precise transport could not be achieved. Photoactuation thus appears as a promising alternative offering contactless, tunable, and highly resolved stimulation. ${ }^{[19,20]}$ However, to our knowledge, only the light induced collapse of photo sensitive particle coated marbles, ${ }^{[10,11]}$ the locomotion of photothermally coated marbles, ${ }^{[21]}$ and the laser guided motion of immersed chloroform marbles by local bubble generation $^{[12]}$ have been reported, each of these approaches being specific to the marble composition. Herein, we describe

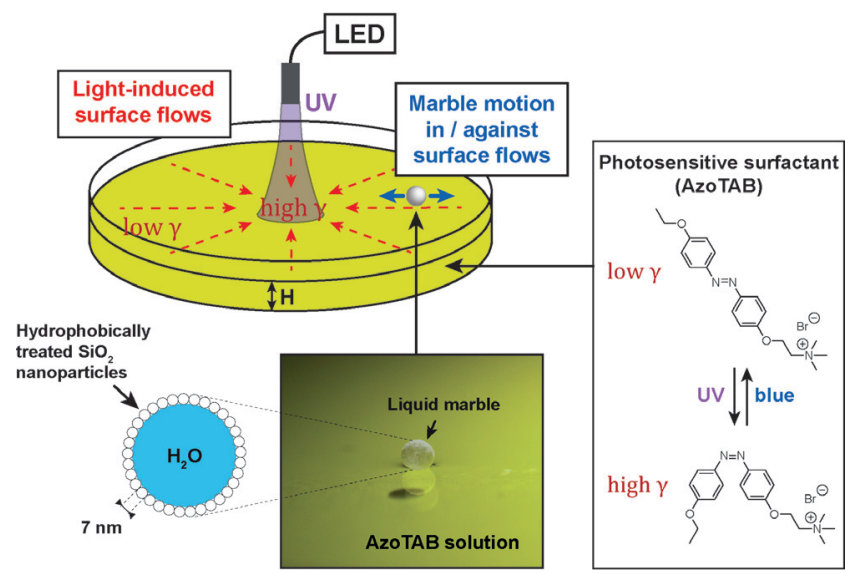

Figure 1. Optical actuation of a floating liquid marble. A $2 \mu \mathrm{L}$ liquid marble is deposited on a water solution containing the surfactant AzoTAB $(10 \mathrm{~mm}$ ) contained within a Petri dish (where $H$ is the liquid thickness). A light guide connected to an LED source provides a Gaussian shaped light spot of width $w$ on the liquid surface, at a wavelength of $\lambda 365 \mathrm{~nm}$ (UV irradiation, $330 \mathrm{Wm}^{2}$ ) or $\lambda \quad 440 \mathrm{~nm}$ (blue light irradiation, $820 \mathrm{Wm}{ }^{2}$ ). The resulting gradient of surface tension $\gamma$ generates light induced surface flows in the direction of the gradient, which, in turn, trigger the motion of the liquid marble either with or against the surface flows. 
for the first time a generic method for remote, long range, and photoreversible transport of floating liquid marbles. To make it applicable regardless of the marble composition, we deposited marbles on a photosensitive surfactant laden solution and used the surface flows driven by the light induced surface tension gradient (Marangoni flows) to actuate the floating marble. We studied the marble motion and discovered a counterintuitive regime where the marble moved in a direction opposite to these surface flows.

We prepared liquid marbles by coating $2 \mu \mathrm{L}$ water drops with hydrophobically modified fumed silica nanoparticles (see Movie S1 in the Support ing Information). Liquid marbles were deposited carefully in an $8.7 \mathrm{~cm}$ diameter Petri dish containing water supplemented by $10 \mathrm{~mm}$ of AzoTAB (Figure 1), a photosensi tive azobenzene containing surfactant. ${ }^{[22,23]}$ Liquid marbles floated on this liquid surface, despite the presence of surfactants and a decreased surface tension $(\gamma$; around $50 \mathrm{mN} \mathrm{m}^{-1}$ ) compared to that of water (see Text S1 in the Supporting Information).

A light guide connected to an LED source was used to irradiate the AzoTAB solution. The light guide was maintained at a fixed distance from the liquid surface and generated a light spot of around $15 \mathrm{~mm}$ in diameter irradiating at $\lambda=365 \mathrm{~nm}$ (UV, $330 \mathrm{~W} \mathrm{~m}^{-2}$ ) or $\lambda=440 \mathrm{~nm}$ (blue, $820 \mathrm{~W} \mathrm{~m}^{-2}$ ). Under these conditions, UV/ blue light irradiation induced a photorever sible increase/decrease of surface tension of $12 \mathrm{mNm}^{-1}$ (Figure S1) due to the photo isomerization of AzoTAB accompanied by an increase/decrease of the polarity of its hydrophobic tail. ${ }^{[24,25]} \mathrm{UV}$ irradiation of the liquid substrate thus induced converging Marangoni surface flows oriented toward the light spot where surface tension was at its maximum. ${ }^{[26,27]}$ The direction of the surface flows was thus independent of the thickness of the liquid substrate layer $H$ (Figure 2a). However, we found that liquid marbles moved in the direction of the surface flow, that is, towards the UV light, only when the value of $H$ was large enough (Figure $2 b$, bottom; Movie S3). Surprisingly, liquid mar bles moved against the direction of surface flow, that is, away from UV light, when the thickness was smaller than a critical value $H^{\text {crit }}$ (Figure 2b top; Movie S2).

When blue light was applied after UV irradiation, the decrease of surface tension in the illuminated area induced diverging Mar angoni flows. These diverging flows also gave rise to two motion regimes. For $H>$ $H^{\text {crit }}$ the marble moved away from the light spot, that is, along the direction of the light induced Marangoni flow, whereas for $H<$
$H^{\text {crit }}$, the marble moved toward the blue light, that is, against the direction of the surface flow. Several cycles of photo reversible motion could be achieved upon successive UV/blue light stimulations for both small and large $H$ values (Fig ure 2c; Movies S4 S5). No marble motion was observed when the same experiment was performed without AzoTAB, showing that light induced Marangoni flows were instrumen tal to the marble motion (Movie S6). To our knowledge, this is the first time that photoreversible transport of a floating liquid marble has been reported. Notably, the marble moved

\section{a) Surface flows}
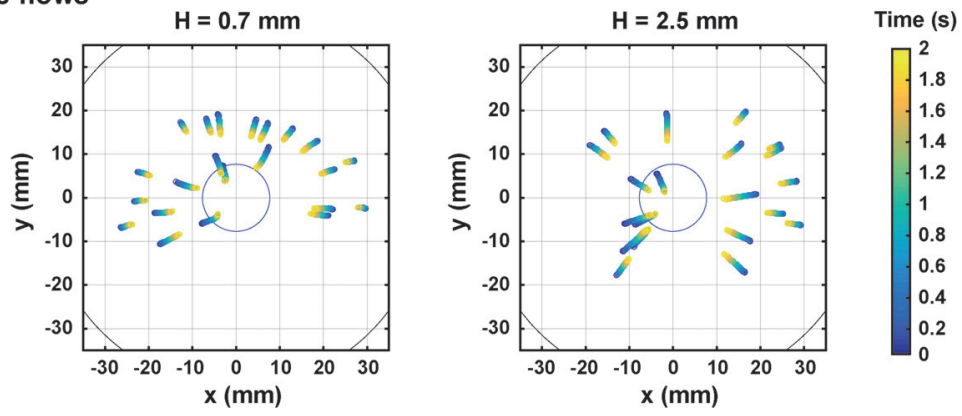

b) Liquid marble motion

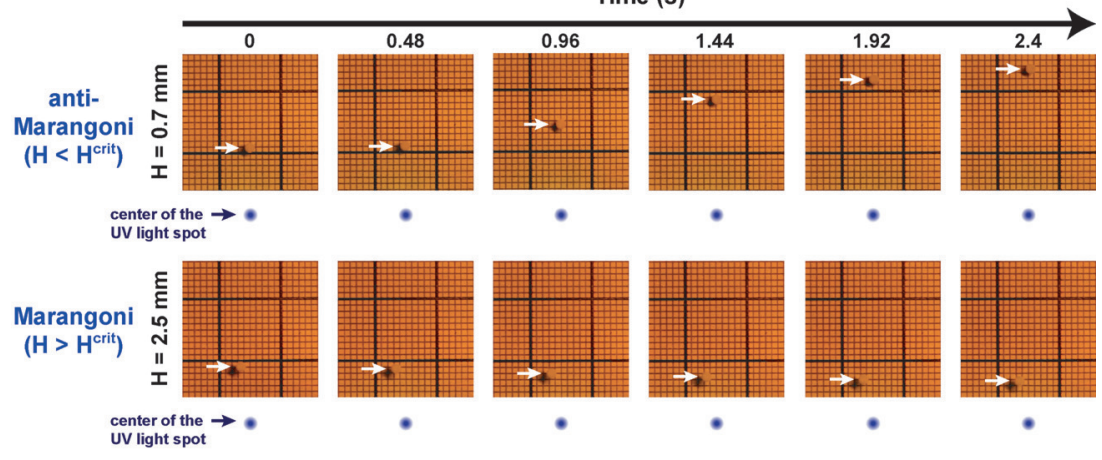

c) Reversible actuation of liquid marbles
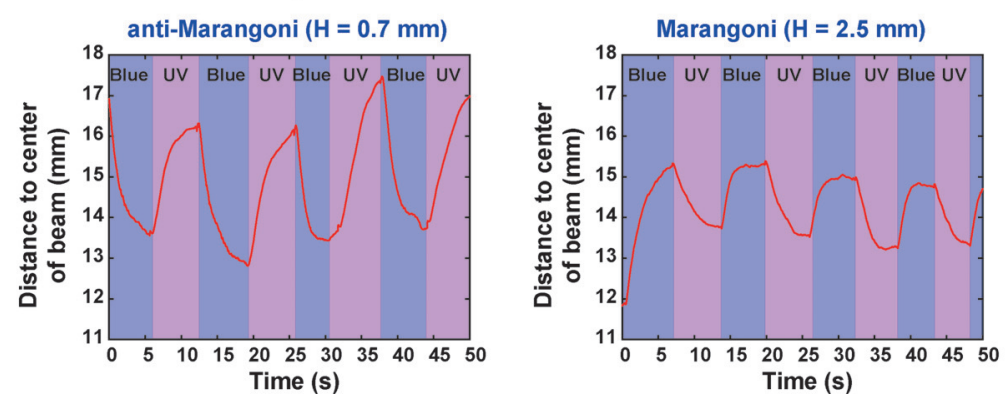

Figure 2. Light induced marble motion is photoreversible and depends on liquid thickness: Marangoni and anti Marangoni motion. a) Trajectories of floating tracers upon UV irradiation as a function of time for two different liquid thicknesses $(H)$. The origin of the $x y$ axes is at the beam center. The purple circle, which has a diameter $2 w$, represents the illuminated zone. The black truncated circle shows the edge of the Petri dish. b) Time lapse photographs of a liquid marble (indicated by a white arrow) upon UV irradiation. The center of the light spot is represented by a purple dot. The marble moves against (anti Marangoni motion, H $\quad 0.7 \mathrm{~mm}$ ) or in the direction (Marangoni motion, $\mathrm{H} \quad 2.5 \mathrm{~mm}$ ) of the surface flow, which is oriented toward the light spot in both cases. The irradiation starts at $t \quad 0$. Corresponding videos are shown in Movie S2 (anti Marangoni regime) and Movie S3 (Marangoni regime). c) Position of the center of a liquid marble as a function of time upon successive blue and UV light irradiation cycles. Corresponding videos are shown in Movie S4 (anti Marangoni) and Movie S5 (Marangoni). 
along (Marangoni motion) or against (anti Marangoni motion) surface flows depending on the thickness of the liquid substrate.

To gain further insight into the origin of this peculiar thickness dependent motion, we investigated the spatiotem poral evolution of liquid marbles actuated with UV light at different distances from the light spot and for various liquid thicknesses (Figure 3a). Regardless of the $H$ value, each marble reacted to the light stimulation with an accelerated motion for about $1 \mathrm{~s}$ followed by a relaxation to a slower and more stable speed. After around $2 \mathrm{~s}$, the fastest moving marbles reached either the edge of the dish or the center of the light spot. For the smallest $H$ values $(H<1.9 \mathrm{~mm})$, the marble was moved away from the UV light source with a speed increasing with decreasing $H$ value, indicating a motion against the light induced Marangoni flows. Note that, in this case, the marble was first attracted to the light spot for a short time (around $0.5 \mathrm{~s}$ ) before being consistently repelled. For intermediate thicknesses $(1.9 \leq H \leq 2.2 \mathrm{~mm})$, the motion was very slow with a speed less than $1 \mathrm{~mm} \mathrm{~s}^{-1}$. For larger liquid layer thicknesses $(H>2.2 \mathrm{~mm})$, the marble moved toward the UV spot, in agreement with Marangoni motion. Interestingly, regardless of the distance of the marble from the illumination spot, the average speed $\left(v^{*}\right)$ taken in the time interval $(0.51 \mathrm{~s})$ decreased with an increase in $H$ value, with an inversion of sign occurring at $H^{\text {crit }} \approx 2.0 \mathrm{~mm}$ (Figure $3 \mathrm{~b}$ ). This value appears to be close to the capillary length of the system $l_{\mathrm{c}}=\sqrt{ } \gamma_{0} /(\rho g)=2.3 \mathrm{~mm}$, where $\gamma_{0}$ is the liquid/air surface tension (without irradiation), $\rho$ is the density of the AzoTAB solution, and $g$ is gravity. The liquid thickness was thus demonstrated to determine the direction of the marble motion with the transition between anti Marangoni and Marangoni regimes defined by a value close to the capillary length.

Although the motion of a floating entity in the direction of a Marangoni flow is commonly observed, ${ }^{[26}{ }^{29]}$ the opposite behavior has been rarely described. ${ }^{[30]}$ When we used a gelled liquid marble, we observed a similar behavior, showing that anti Marangoni motion was not due to flow inside the marble (see Text S2 in the Supporting Information). Moreover, by closely examining the behavior of the marble upon its displacement, although some slow rotation around the vertical axis was occasionally observed, there was no signifi cant rotation in the direction of motion. We therefore hypothesized that the Marangoni flow induced deformation of the AzoTAB solution surface could strongly affect the marble motion behavior and explain, in particular, the anti Marangoni flow motion.

To explore this hypothesis, we used a custom built set up to estimate, using a reflected laser beam, the surface deformation over time upon light actuation (Figure S2). Strikingly, when the UV light was on, although no significant deformation (typically less than $10 \mu \mathrm{m}$ ) could be detected for a large liquid layer thickness $(H=2.5 \mathrm{~mm})$, we measured a difference in surface elevation between the edge of the Petri dish and the center of the beam of up to $180 \mu \mathrm{m}$ at low thickness $(H=0.7 \mathrm{~mm}$; Figure $4 \mathrm{a})$. In the latter case, the converging Marangoni flow thus accumulated liquid at the beam center deforming the surface to create a slope, so that a)
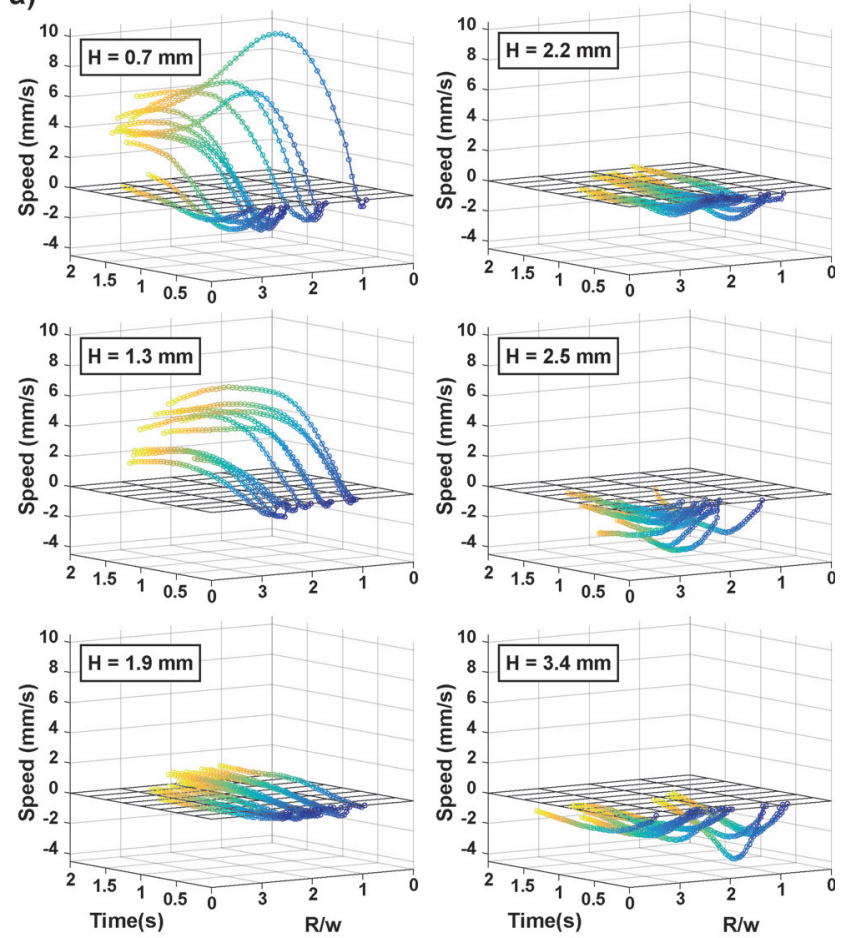

b)

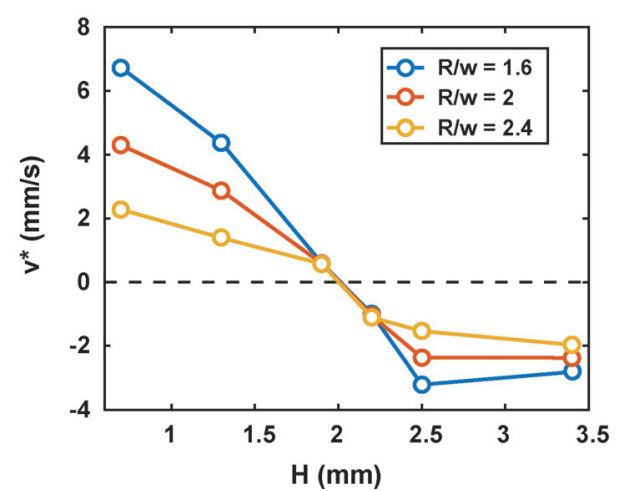

Figure 3. Spatiotemporal analysis of the liquid marble motion shows that the transition between Marangoni and anti Marangoni regimes occurs at $H \quad 2 \mathrm{~mm}$. a) Radial speed of a liquid marble upon UV irradiation as a function of time and initial distance to the beam center $(R)$ normalized by the beam width $(w)$, for various liquid thicknesses $(H)$. UV irradiation starts at $t \quad 0$. Lines connecting points are drawn as guides to the eye and indicate the trajectory of a given marble. Marangoni flows are oriented toward the beam center, with a positive or negative speed indicating anti Marangoni or Marangoni behavior, respectively. b) Average speed of the marble ( $\left.v^{*}\right)$ taken in the interval $0.51 \mathrm{~s}$ as a function of $H$ value for different values of $R / w$. The horizontal dashed line indicates $v^{*} 0$.

a liquid marble deposited on this system slid against the flow under the effect of gravity. We call this behavior a "slide effect". The slope formation required around $3 \mathrm{~s}$, which was longer than the observation time of marble motion (Fig ure 3 a), showing that although a deformation steady state was not attained in our marble motion experiments, the forming slope was steep enough to propel the marble against the surface flow. It should also be noted that for the larger distances from the beam center, no deformation was observed 


\section{a) Surface deformation}
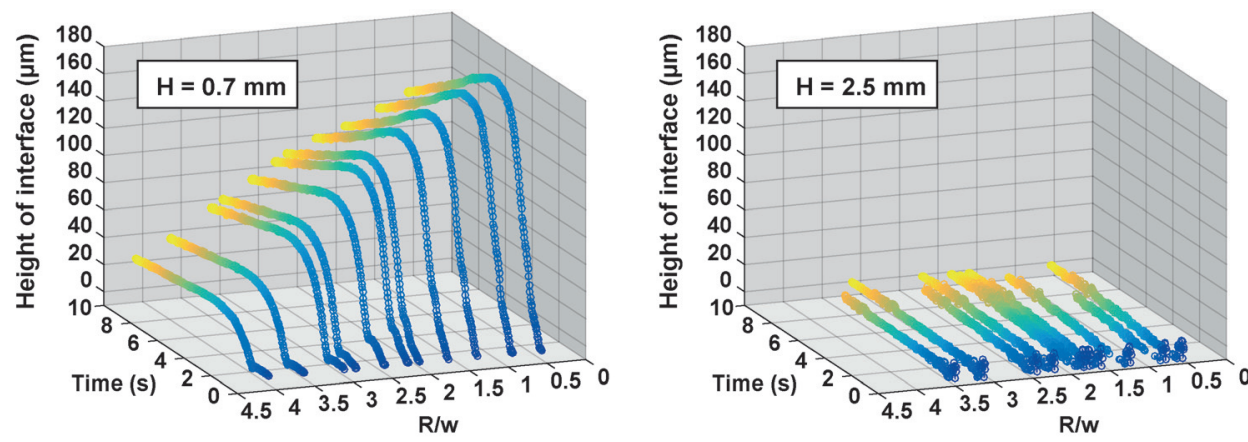

b) Mechanism
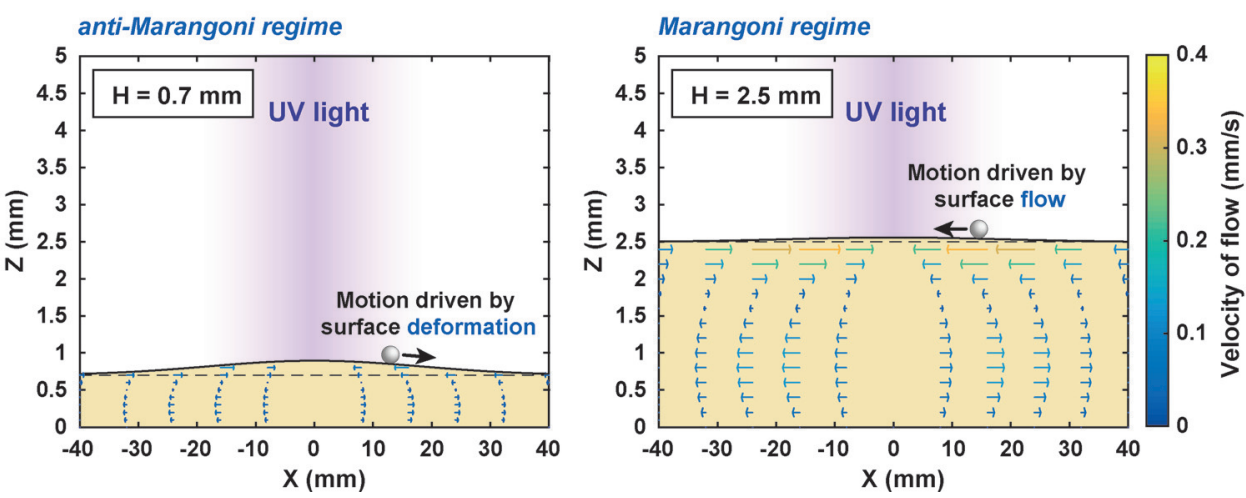

Figure 4. Experiments and theory show that light induced surface deformation depends on liquid thickness and defines the motion regime. a) Measured surface elevation (fixed at zero at the edge of Petri dish) upon UV irradiation as a function of time and distance to the beam center $(R)$ normalized by the beam width $(w)$, for two liquid thicknesses $\left(\begin{array}{ll}H & 0.7 \text { and } 2.5 \mathrm{~mm}\end{array}\right)$. UV irradiation starts at $t \quad 0$. Lines connecting points are guides for the eye and indicate the evolution of the surface at a given distance $R / w$. b) Illustration of the mechanism for both anti Marangoni (left) and Marangoni (right) regimes. In each graph, the surface shape is a Gaussian fit from the measured deformation averaged in the time interval $36 \mathrm{~s}$. The flow profile, indicated by colored arrows, was calculated by our analytical model (see Text S4 in the Supporting Information). For $H \quad 0.7 \mathrm{~mm}$, light induced surface flows induce a significant deformation that propels the marble upstream, whereas for $H \quad 2.5 \mathrm{~mm}$, deformation is negligible and the marble moves in the direction of the strong surface flows.

for about $0.5 \mathrm{~s}$, corresponding well with the observation that at $H<H^{\text {crit }}$, the marble was moving first, for a short time, in the direction of the Marangoni flows before being propelled in the opposite direction (anti Marangoni motion; Figure $3 \mathrm{a}$ ). All of these observations show that the marble moved in the direction of the Marangoni flows when surface deformation was negligible (that is, at $t=00.5 \mathrm{~s}$ for $H<H^{\text {crit }}$ and regard less of time for $H>H^{\text {crit }}$ ), and in the opposite direction when surface deformation was strong enough (that is, at $t>0.5 \mathrm{~s}$ for $\left.H<H^{\text {crit }}\right)$.

To evaluate the relevance of this surface deformation dependent motion behavior, we built an analytical hydro dynamic model where we derived the velocity profile of the Marangoni flow and the resulting surface deformation (Text S3). To identify the underlying mechanism at the origin of the marble motion, the calculation was carried out in a stationary regime so that analytical solutions could be found to extract essential physical parameters (although dynamic effects, as described previously, are obviously present in this system). When the capillary length $\left(l_{\mathrm{c}}\right)$ is smaller than the beam width ( $w$; under our experimental conditions, $l_{\mathrm{c}}=2.3 \mathrm{~mm}$ and $w$ $\approx 7.5 \mathrm{~mm}$ ), we found a simple expression that describes the local variation of the interface height:

$\delta h(r) \quad \frac{3}{2 \rho g H}\left(\gamma(r) \quad \gamma_{0}\right)$

where $\gamma_{0}$ is the surface tension in the absence of irradiation, $\gamma$ is the surface tension upon irradi ation, and $r$ is the distance from the beam center. This expression shows that, regardless of $H$ value, the UV induced increase of sur face tension is accompanied by an elevation of the surface, in agreement with the observed sur face elevation in the illuminated zone. Moreover, Equation (1) emphasizes the strong effect of the liquid thickness in converting a surface tension gradient into surface deformation: the eleva tion increases with a decrease in $H$ value, a behavior that was also experimentally observed (Fig ure 4a). By making a force bal ance on the floating marble taking into account drag force, weight, buoyancy, and surface tension, we also calculated the speed of the marble $V$ as a func tion of the distance from the beam center $(r)$ and liquid layer thickness (see Text S3 in the Supporting Information):

$V(r) \quad \frac{H}{4 \eta}\left[1 \quad\left(\frac{A l_{c}}{H}\right)^{2}\right] \partial_{r} \gamma$

where $\eta$ is the liquid substrate viscosity and $A$ is a dimension less parameter related to the geometry of the system expected to be of the order of 1 . Recalling that upon UV actuation $\partial_{r} \gamma \quad 0$ as surface tension is at a maximum at the center of the beam, this equation thus states that if $H \gtrsim l_{\mathrm{c}}$, the marble is dragged by the surface flow toward the beam center (Marangoni motion) but, as soon as $H \lesssim l_{\mathrm{c}}$, the direction of the motion is reversed and the liquid marble slides upstream (anti Marangoni motion). This is in remarkable agreement with our experimental results where the observed reversal of motion occurred at $H^{\text {crit }} \approx 2.0 \mathrm{~mm}$ (Figure $3 \mathrm{~b}$ ), a value close to the capillary length.

Based on our experimental and theoretical analyses, we can thus propose a mechanism explaining both the Marangoni and anti Marangoni regimes. Figure $4 \mathrm{~b}$ shows the behavior of the marble for each regime at $H=0.7 \mathrm{~mm}$ (anti Marangoni) and at $H=2.5 \mathrm{~mm}$ (Marangoni) under stationary conditions. 
The plotted surface deformation is a Gaussian fit of the measured deformation averaged over the time interval ( 3 $6 \mathrm{~s})$, which was also used to estimate $\gamma(r)$ using Equation (1). According to this estimate, the $\gamma$ value was found to increase by $1.0 \mathrm{mN} \mathrm{m}^{-1}$ between the edge and the center of the Petri dish, which is in good agreement with the measured increase of $\gamma$ upon UV irradiation (Figure S1). The flow profile was then calculated using our analytical model and this estimation of $\gamma(r)$ (Text S4). Figure 4b shows that in both anti Marangoni and Marangoni regimes, the converging surface flow results in a recirculation of the liquid in the bulk, which can occur more easily at a larger thickness. Interestingly, although the speed of the surface flow decreases with a decrease in $H$ value, the resulting surface deformation increases due the confinement of the liquid. This results in i) a strong deformation propelling the marble upstream at low $H$ value (anti Marangoni regime) and ii) a strong surface flow with negligible surface deformation resulting in downstream marble motion at high $H$ value (Marangoni regime).

In summary, we have shown that liquid marbles can be actuated by light by employing a liquid substrate with a photosensitive liquid/air surface tension. This is achieved by using a photosensitive surfactant dissolved in the liquid substrate, but other ways to trigger a surface tension change could be used, such as local heating upon laser illumination. For a large liquid thickness, the marble is transported by surface Marangoni flows that can be photoreversibly trig gered upon UV/blue light irradiation (Marangoni motion). Remarkably, we have discovered that at a liquid thickness smaller than a value close to the capillary length of the liquid substrate, the marble moves in the opposite direction (anti Marangoni motion). We have shown that, in this case, light induced surface flows create a slope in the liquid substrate so that gravity propels the marble upstream, a behavior we term the "slide effect". These results emphasize how well known surface flows ${ }^{[31,32]}$ can be employed as useful operators for the precise actuation of miniature systems.

\section{Acknowledgements}

This work was supported by the Mairie de Paris (Emergence (s) 2012). M.A. acknowledges funding from the European Commission (FP7 PEOPLE 2013 IEF/Project 624806 "DIOPTRA"). N.K. thanks Maria Vladimirova (L2C, CNRS) for help with data analysis and Alexey Kavokin (University of Southampton) for discussion.

Keywords: interfaces · liquid marbles - Marangoni flows · photoactuation · surface tension
[2] D. C. Duffy, J. C. McDonald, O. J. A. Schueller, G. M. White sides, Anal. Chem. 1998, 70, 49744984.

[3] R. B. Fair, Microfluid. Nanofluid. 2007, 3, 245281.

[4] M. K. Chaudhury, G. M. Whitesides, Science 1992, 256, 1539 1541.

[5] K. Ichimura, S. K. Oh, M. Nakagawa, Science 2000, 288, 1624 1626.

[6] S. Daniel, M. K. Chaudhury, J. C. Chen, Science 2001, 291, 633 636.

[7] P. Aussillous, D. Quéré, Nature 2001, 411, 924927.

[8] P. Aussillous, D. Quéré, Proc. R. Soc. London Ser. A 2006, 462, 973999.

[9] G. McHale, M. I. Newton, Soft Matter 2015, 11, 25302546.

[10] C. H. Ooi, N. T. Nguyen, Microfluid. Nanofluid. 2015, 19, 483 495.

[11] K. Nakai, S. Fujii, Y. Nakamura, S. Yusa, Chem. Lett. 2013, 42, 586588.

[12] Y. Chu, F. Liu, L. Qin, Q. Pan, ACS Appl. Mater. Interfaces 2016, $8,12731279$.

[13] J. R. Dorvee, A. M. Derfus, S. N. Bhatia, M. J. Sailor, Nat. Mater. 2004, 3, 896899.

[14] E. Bormashenko, R. Pogreb, Y. Bormashenko, A. Musin, T. Stein, Langmuir 2008, 24, 1211912122.

[15] Y. Zhao, Z. Xu, M. Parhizkar, J. Fang, X. Wang, T. Lin, Microfluid. Nanofluid. 2012, 13, 555564.

[16] M. I. Newton, D. L. Herbertson, S. J. Elliott, N. J. Shirtcliffe, G. McHale, J. Phys. D 2007, 40, 2024.

[17] E. Bormashenko, R. Pogreb, R. Balter, O. Gendelman, D. Aurbach, Appl. Phys. Lett. 2012, 100, 151601.

[18] D. Zang, J. Li, Z. Chen, Z. Zhai, X. Geng, B. P. Binks, Langmuir 2015, 31, 1150211507.

[19] D. Baigl, Lab Chip 2012, 12, 36373653.

[20] D. Okawa, S. J. Pastine, A. Zettl, J. M. J. Fréchet, J. Am. Chem. Soc. 2009, 131, 53965398.

[21] M. Paven, H. Mayama, T. Sekido, H. Butt, Y. Nakamura, S. Fujii, Adv. Funct. Mater. 2016, 26, 31993206.

[22] A. Diguet, R. M. Guillermic, N. Magome, A. Saint Jalmes, Y. Chen, K. Yoshikawa, D. Baigl, Angew. Chem. Int. Ed. 2009, 48, 9281 9284; Angew. Chem. 2009, 121, 94459448.

[23] A. Venancio Marques, F. Barbaud, D. Baigl, J. Am. Chem. Soc. 2013, 135, 32183223.

[24] A. Diguet, N. K. Mani, M. Geoffroy, M. Sollogoub, D. Baigl, Chem. Eur. J. 2010, 16, 1189011896.

[25] E. Chevallier, A. Mamane, H. A. Stone, C. Tribet, F. Lequeux, C. Monteux, Soft Matter 2011, 7, 78667874.

[26] S. N. Varanakkottu, S. D. George, T. Baier, S. Hardt, M. Ewald, M. Biesalski, Angew. Chem. Int. Ed. 2013, 52, 7291 7295; Angew. Chem. 2013, 125, 74327436.

[27] A. Venancio Marques, D. Baigl, Langmuir 2014, 30, 42074212.

[28] E. Bormashenko, Y. Bormashenko, R. Grynyov, H. Aharoni, G. Whyman, B. P. Binks, J. Phys. Chem. C 2015, 119, 99109915.

[29] C. H. Ooi, A. van Nguyen, G. M. Evans, O. Gendelman, E. Bormashenko, N. T. Nguyen, RSC Adv. 2015, 5, 101006101012.

[30] E. Yakhshi Tafti, H. J. Cho, R. Kumar, Appl. Phys. Lett. 2010, 96 , 264101.

[31] J. W. M. Bush, D. L. Hu, Annu. Rev. Fluid Mech. 2006, 38, 339 369.

[32] N. J. Cira, A. Benusiglio, M. Prakash, Nature 2015, 519, 446450.

[1] G. M. Whitesides, Nature 2006, 442, 368373. 